\title{
Power Transmission of UHF Passive Embedded RFID in Tires
}

\author{
Shengbo Hu, ${ }^{1,2}$ Bing Si, ${ }^{3}$ Heng Shu, ${ }^{1,2}$ and Jinrong Mo ${ }^{1,2}$ \\ ${ }^{1}$ Institute of Intelligent Information, Guizhou Normal University, Guiyang 550001, China \\ ${ }^{2}$ Department of Guizhou Education, Center for RFID and WSN Engineering, Guiyang 550001, China \\ ${ }^{3}$ Institute of New Technology, Guizhou Academy of Sciences, Guiyang 550001, China \\ Correspondence should be addressed to Shengbo Hu; hsb@nssc.ac.cn
}

Received 5 August 2013; Revised 28 December 2013; Accepted 30 December 2013; Published 17 February 2014

Academic Editor: Yuan Yao

Copyright (C) 2014 Shengbo Hu et al. This is an open access article distributed under the Creative Commons Attribution License, which permits unrestricted use, distribution, and reproduction in any medium, provided the original work is properly cited.

\begin{abstract}
UHF passive RFID tags embedded in tires have a deep impact on tire life cycle management and tire monitoring. In this work, we present the power transmission of UHF passive embedded RFID in tires. In UHF passive embedded RFID systems in tires, the bidirectional radio link between reader and tags goes through air and tires. The total path loss contains reflection loss at tire-air boundaries and attenuation loss in the tires. The power transmission is based on the permittivity of tires and tire-air boundary conditions. We give an OCP method for measuring the permittivity of tires. By analyzing the radio link for UHF passive RFID, we establish a model of wave propagation of UHF embedded RFID in tires and make numerical analyses. Numerical analyses show that the error of the OCP methods for measuring the permittivity of tires is small, the parallel polarization and normal incidence of wave are chosen for improving the performance of the UHF embedded RFID in tires, and distance is chosen to keep power transmission function from locating valley.
\end{abstract}

\section{Introduction}

Acting as sensors, passive RFID tags can avoid sensor nodes bulky and battery powered. For that reason, UHF passive tags embedded in tires have been used widely for tire life cycle management in the USA and the European Union $[1,2]$. To meet the Automotive Industry Action Group's B-11 standard for North American, Michelin began offering automakers the option of purchasing tires with embedded tags [3]. Besides, the combinations of UHF passive tags embedded in tire and tire pressure monitoring are paid attention highly to improve the reliability of tire and tire control systems [4].

However, range has been one of the hardest challenges in UHF passive RFID embedded in tires, because the rubber makes it harder to read the tag. When Michelin took offthe-shelf, UHF passive tags and embedded them in tires, the read distance dropped to less than three inches [3]. The main difference between the common RFID and RFID embedded in tires is communication medium, which attenuates RF power from the reader in RFID embedded in tires. To improve the range and reliability of RFID embedded in tires, it is of great concern to study power transmission of wave propagation for UHF passive embedded RFID in tires, because the tags do not contain any battery and rely on the electromagnetic field for both power and communication. In this paper, we present the power transmission of wave propagation for UHF passive embedded RFID in tires and lay out the foundations for reliable communication in this environment.

In UHF passive RFID systems, a bidirectional radio link is established between reader and tags, which can be classified a forward link from the reader to tags and a backward link from the tags to reader [5]. Depending on the characteristics of reader and tags, the propagation channel properties like path loss and fading, the power transmission coefficient and the channel transmissions are investigated in [5-7]. In UHF passive embedded RFID systems in tires, the bidirectional radio link between reader and tags goes through air and tires. The total path loss contains several factors: reflection loss due to reflected power at tire-air boundaries, attenuation loss in the tires, and spreading loss which is simply due to the radiation properties of antenna. Each of these factors can be analyzed using the permittivity of tires and tire-air boundary conditions. 


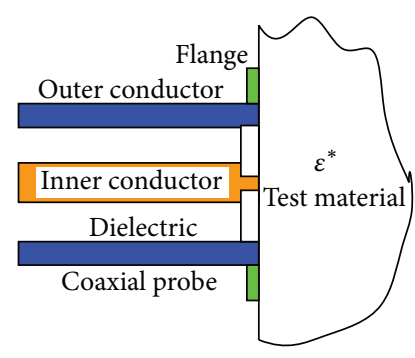

(a) Measuring system

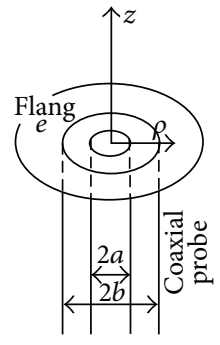

(b) Geometry model

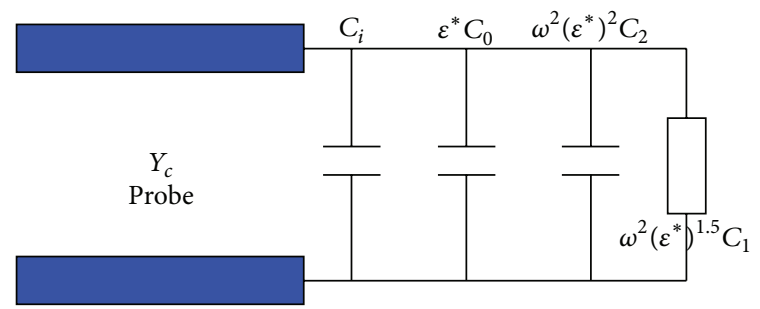

FIgURE 2: The equivalent circuit of admittance model for a coaxial open ended probe.

FIGURE 1: Measuring reflection coefficients with open ended coaxial probe.

So, this paper focuses on wave propagation for UHF passive embedded RFID in tires based on the permittivity of tires and tire-air boundary conditions. Hence, Section 2 presents how to measure the permittivity of tires. Section 3 describes the radio link for UHF RFID systems. Section 4 gives a propagation model of UHF passive embedded RFID systems in car tires. Section 5 describes numerical calculation and discussion. Section 6 concludes this paper.

\section{Measuring the Permittivity of Tires}

2.1. Measuring the Permittivity. Several techniques have been developed for measuring the permittivity [8-10]. However, some, such as resonant cavity or wave-guide transmission line cells, require test hardware machining and destructive processing. And open ended coaxial probe (OCP) technique is currently one of the most popular techniques for measuring the permittivity. OCP technique can perform nondestructive, broadband (RF and microwave bands), and high temperature measurement. Its well-developed theory makes it possible to obtain sufficiently accurate results.

A schematic for a coaxial open ended probe is shown in Figure 1. To reduce significantly measuring error, the material being tested is placed in close contact with the probe's flat end. The reflection coefficient, measured with a vector network analyzer (VNA), is used as inverse calculation of the permittivity of the material.

An equivalent circuit of admittance model for a coaxial open ended probe is shown in Figure 2. In Figure 2, $Y_{c}$ is the probe characteristic admittance, and $Y\left(\omega, \varepsilon^{*}\right)$ is the load admittance, including the inner admittance $Y_{i}(\omega)=j \omega C_{i}$ of the capacitance $C_{i}$ between outer conductor and inner conductor and the outer admittance $Y_{o}\left(\omega, \varepsilon^{*}\right)$, obtained by solving the following equation [11]:

$$
Y_{o}\left(\omega, \varepsilon^{*}\right)=j \frac{2 \omega \varepsilon_{0} \varepsilon^{*}}{[\ln (b / a)]^{2}} \iint_{a}^{b} \int_{0}^{\pi} \cos \varphi \frac{e^{-j k r}}{r} d \varphi d \rho^{\prime} d \rho,
$$

where $k=\omega \sqrt{\mu \varepsilon^{\prime}}, \mu$ is the permeability, $\omega$ is the operating frequency, $\left(\rho, \rho^{\prime}, \varphi\right)$ are cylindrical coordinates, $r=$ $\left[\rho^{2}+\rho^{\prime 2}-2 \rho \rho^{\prime} \cos \varphi\right]^{1 / 2}$, and $a$ and $b$ are, respectively, the interior and the external radius of the probe. And (1) can be the series expansion form of (2):

$$
Y_{o}\left(\omega, \varepsilon^{*}\right)=\sum_{n=0}^{\infty}(-1)(-j \omega)^{n+1} C_{n} \varepsilon^{*(n+2) / 2},
$$

where

$$
C_{n}=\frac{2\left(\mu_{0} \varepsilon_{0}\right)^{(n+2) / 2}}{n ! \mu_{0}[\ln (b / a)]^{2}} \iint_{a}^{b} \int_{0}^{\pi} r^{n-1} \cos \varphi d \varphi d \rho^{\prime} d \rho .
$$

Equation (3) shows that $C_{n}$ is constant. For convenience, (2) can be truncated to 3 terms with the $C_{0}, C_{1}$, and $C_{2}$. So, $Y\left(\omega, \varepsilon^{*}\right)$ can be given as below:

$$
Y\left(\omega, \varepsilon^{*}\right)=j \omega C_{i}+j \omega \varepsilon^{*} C_{0}+\omega^{2}\left(\varepsilon^{*}\right)^{1.5} C_{1}+j \omega^{3}\left(\varepsilon^{*}\right)^{2} C_{2},
$$

where $\varepsilon^{*} C_{0}$ is the fringing capacitance of the probe, $\omega^{2}\left(\varepsilon^{*}\right)^{2} C_{2}$ is the fringing capacitance generated from a test material, and $\omega^{2}\left(\varepsilon^{*}\right)^{1.5} C_{1}$ is the equivalent conductance radiated from the probe.

The load admittance $Y\left(\omega, \varepsilon^{*}\right)$ can be obtained from the EM wave reflection coefficient $\Gamma\left(\omega, \varepsilon^{*}\right)$ using the following relation:

$$
Y\left(\omega, \varepsilon^{*}\right)=Y_{c} \frac{1-\Gamma\left(\omega, \varepsilon^{*}\right)}{1+\Gamma\left(\omega, \varepsilon^{*}\right)}
$$

Hence, measuring the permittivity can be performed using the following steps.

Step 1. Using VNA, we measure the four different reflection coefficients $\Gamma_{1}, \Gamma_{2}, \Gamma_{3}$, and $\Gamma_{4}$ associated with the four different frequencies using a standard material with the known permittivity. Using (4) and (5), the four unknown parameters $C_{i}$, $C_{0}, C_{1}$, and $C_{2}$ can be solved.

Step 2. Using VNA, we measure the reflection coefficients $\Gamma$ associated with some frequencies using the test material. Using (4) and (5), the permittivity of the test material can be calculated by a suitable iterative method.

2.2. Measurement Setup for Tires. The tire sidewall and the position of the RFID tag embedded in tire are displayed in Figure 3. The tag is embedded parallel to the outer steel mesh 

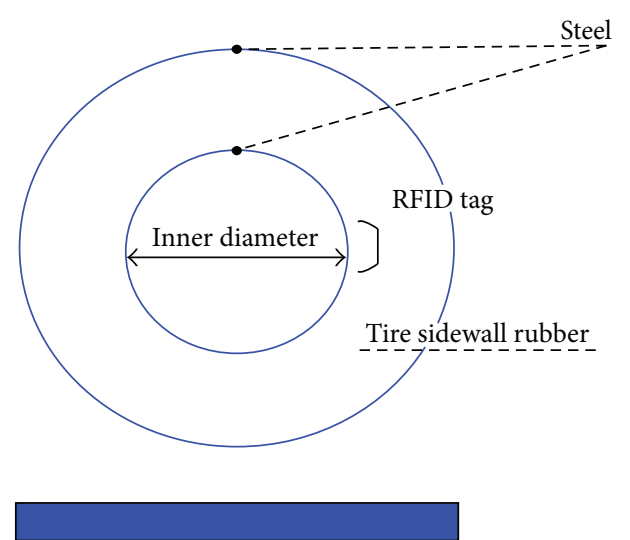

FIgURE 3: The RFID tag embedded in tires.

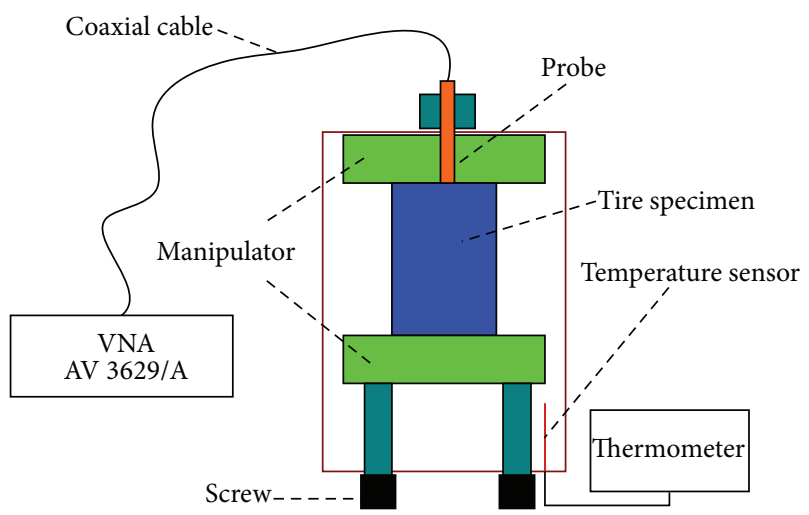

Figure 4: The OCP measurement system.

at a distance that depends on the tire size and ranges from 4 to $8 \mathrm{~cm}$ above the inner steel mesh [1].

To reduce significantly measuring error using OCP technique, the tire sidewall specimen has to meet the following conditions.

(i) The surface of the tire sidewall specimen has to be flat. A piece of the tire sidewall is cut out, and the cross section is polished to obtain a flat surface.

(ii) The specimen has to have semi-infinite thickness so that the penetration of the field must be much smaller than the specimen's thickness.

(iii) There must not be air gaps between probe and the tire sidewall specimen.

Figure 4 illustrates an OCP measurement system. The system consists of a VNA, a thermometer, a coaxial probe, and a manipulator with screw for fixing the tire sidewall specimen. Tightening the screw, the probe is pressed against the specimen until no more variation of the measurement results is observed.

2.3. Simulation Verifying Using HFSS. Simulation verifying using HFSS to verify the measurement accuracy using OCP technique, an OCP model using HFSS (high frequency

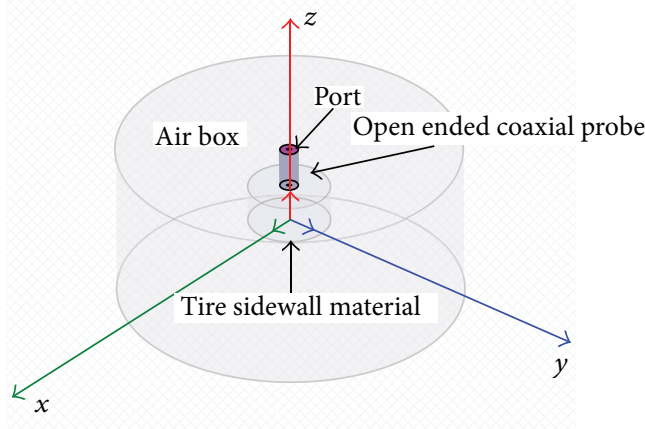

FIGURE 5: OCP model based on HFSS.

structure simulator) is set up to compare. Given the measured permittivity of tire specimen using the measuring methods presented in Section 2.1, the reflection coefficient is solved using the OCP model. And the simulation and measurement reflection coefficients are compared.

The OCP model based on HFSS is shown in Figure 5. In Figure 5, the inner and outer conductors of the probe are made of gilded brass, and the space between the inner and outer conductor is filled in Teflon dielectric material. The tire specimen is modeled as a cylinder. To avoid perfectly conducting boundaries around the tire sidewall material, an air box is implemented that surrounds the whole simulation setup.

\section{Radio Link for UHF Passive RFID}

In passive UHF RFID systems, a bidirectional radio link is established between reader and tags, including a forward link from the reader to tags and a backward link from the tags to reader. What radio link for UHF passive RFID differs from conventional radio is that the backward link resembles radar link. Besides, the RFID tag's antenna absorbs waves as a function of its load match and reemits waves as a function of its load mismatch.

3.1. Power Absorbing Coefficient. RFID tag consists of an antenna and chip. The impedance matching of tag antenna and chip strongly influences the RF power transmission and the communication performance between reader and tags. Because of IC technological limit, the impedance of a chip cannot be chosen arbitrarily [12]. So, the chip's input impedance is designed to switch between two values, between the conjugate impedance called as absorbing impedance $Z_{\text {chip1 }}$ and other impedance called as reflecting impedance $Z_{\text {chip2 }}$. The quality of the impedance match can be defined by the power absorbing coefficient $\tau$, which is the power accepted by the chip and the available power $P_{\text {tag }}$ at the antenna port $[5,12]$ :

$$
\tau=\frac{4 \operatorname{Re}\left[Z_{\text {ant }}\right] \operatorname{Re}\left[Z_{\text {chipl }}\right]}{\operatorname{Re}\left[Z_{\text {ant }}+Z_{\text {chipl } 1}\right]^{2}+\operatorname{Im}\left[Z_{\text {ant }}+Z_{\text {chip } 1}\right]^{2}},
$$


where $Z_{\text {ant }}$ is the tag antenna's input impedance and $0 \leq \tau \leq$ 1. When $\tau=1$, all available power is absorbed by the chip. And when $\tau=0$, no available power is absorbed by the chip. So, the range of frequency that $\tau$ reaches a demand value is defined as the bandwidth of the tag antenna. This shows that a best designed tag antenna is very important to improve the performance of UHF passive RFID.

3.2. Power Transmission Coefficient. In a passive RFID, the power absorbed by the chip in forward link can be expressed as

$$
P_{\text {chip }}=\tau P_{\text {tag }}=\tau\left|S_{21}\right|^{2} P_{\mathrm{TX} . \text { reader }}
$$

where $P_{\text {TX.reader }}$ is the transmitted power from reader and $P_{\text {chip }}$ must be higher than the tag's sensitivity for the tag's normal work. And $\left|S_{21}\right|^{2}$ is the power transmitted coefficient in the forward link, depending on the antenna power transmission of reader and tag (e.g., antenna gain) and the propagation channel properties like path loss and fading [5].

The power received by the reader from the tag can be expressed as in the backward link:

$$
P_{\text {RX.reader }}=\left|S_{12}\right|^{2} \eta P_{\text {tag }}=\left|S_{12}\right|^{2} \eta\left|S_{21}\right|^{2} P_{\text {TX.reader }},
$$

where $\eta$ is the modulation efficiency, which is defined as the ratio of the power scattered by the tag and the power available at the tag antenna output. $\left|S_{12}\right|^{2}$ is the power transmitted coefficient in the backward link. As real propagation environment is symmetrical, $\left|S_{12}\right|^{2}=\left|S_{21}\right|^{2}$.

\section{Model of Power Transmission of UHF Passive RFID Embedded in Tires}

4.1. Harmonic Waves. For time-harmonic fields, when the medium presents a conductivity $\sigma$ and permeability $\mu$, at the operating frequency $\omega$, a permittivity $\varepsilon^{*}$, the wave equation can be written as a time-independent wave equation towards $+z$ direction:

$$
\begin{gathered}
\nabla^{2} E-\gamma^{2} E=0, \\
\nabla^{2} H-\gamma^{2} H=0, \\
-\gamma^{2}=k^{2}(1-j \tan \delta)=\omega^{2} \mu \varepsilon^{*},
\end{gathered}
$$

where $\varepsilon^{*}=\varepsilon^{\prime}(1-j \tan \delta)$ and $k=\omega \sqrt{\mu \varepsilon^{\prime}}$ is the wave number corresponding to an unbounded lossless medium with a real dielectric constant $\varepsilon^{\prime}$.

For uniform plane waves, $\nabla^{2}=\partial^{2} / \partial^{2} z$, so (9) and (10) have solutions of the form $E e^{\gamma z}$ and $H e^{\gamma z}$, and the instantaneous values for the fields are given as follows:

$$
\begin{aligned}
& \vec{E}=\operatorname{Re}\left\{E e^{-\alpha z} e^{j(\omega t-\beta z)}\right\}, \\
& \vec{H}=\operatorname{Re}\left\{H e^{-\alpha z} e^{j(\omega t-\beta z)}\right\},
\end{aligned}
$$

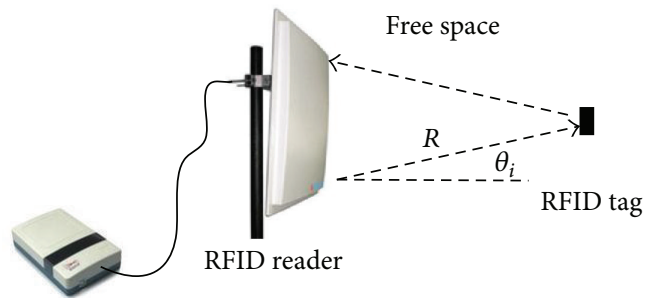

(a) RFID scenarios in free space

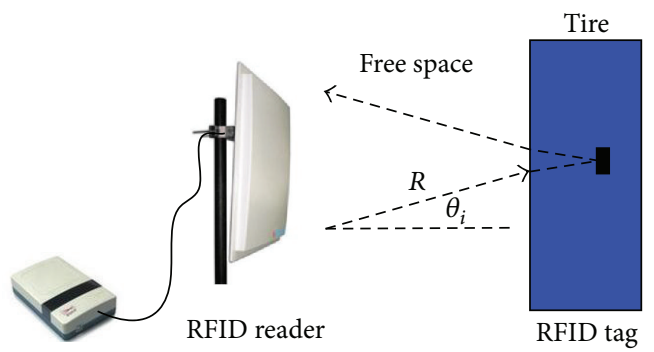

(b) RFID scenarios embedded in tire

FIGURE 6: RFID scenarios.

where the attenuation constant $\alpha$ and the phase constant $\beta$ can be expressed respectively as follows [13]:

$$
\begin{aligned}
& \alpha=\omega\left(\frac{\mu \varepsilon^{\prime}}{2}\right)^{1 / 2}\left[\left(1+\tan ^{2} \delta\right)^{1 / 2}-1\right]^{1 / 2}, \\
& \beta=\omega\left(\frac{\mu \varepsilon^{\prime}}{2}\right)^{1 / 2}\left[\left(1+\tan ^{2} \delta\right)^{1 / 2}+1\right]^{1 / 2} .
\end{aligned}
$$

So, the electric strength decreases with distance $z$ are given as:

$$
E=E_{0} e^{-\alpha z} \cos \beta z
$$

Substituting (14) into power equation, we have

$$
P=P_{0}|\cos 2 \beta z| e^{-2 \alpha z}
$$

4.2. Power Transmission for RFID Embedded in Tires. The RFID scenarios in space and embedded in tires are shown in Figure 6. In Figure 6, the incident angle of waves is $\theta_{i}$.

In the free space, the absorbing power by the RFID tag is given as

$$
\begin{aligned}
P_{\text {chip }} & =\tau P_{\text {tag }}=\tau\left|S_{21}\right|^{2} P_{\text {TX.reader }} \\
& =\tau\left(\frac{\lambda}{4 \pi R}\right)^{2} G_{T} P_{\text {TX.reader }},
\end{aligned}
$$

where $\lambda$ is the free space wavelength, $R$ is the distance from RFID reader, and $G_{T}$ is the gain of the tag's antenna. 
In the scenarios embedded in tires, (16) can be modified as

$$
\begin{aligned}
P_{\text {chip }} & =\tau P_{\text {tag }}=\tau\left|S_{21}\right|^{2} P_{\mathrm{TX} . \text { reader }} \\
& =\tau\left(\frac{\lambda}{4 \pi R_{1}}\right)^{2}\left|T_{c}\right|^{2} G_{T}|\cos 2 \beta l| e^{-2 \alpha l} P_{\mathrm{TX} . \text { reader }} \\
& =\tau\left(\frac{\lambda}{4 \pi R_{1}}\right)^{2} G_{t} T\left(f, \theta_{i}, l\right) P_{\mathrm{TX} . \text { reader }},
\end{aligned}
$$

where $R_{1}$ is the distance between tires and the reader, $T_{c}$ is electric field transmission coefficient at boundary of tires and free space, and $T\left(f, \theta_{i}, l\right)=\left|T_{c}\right|^{2}|\cos 2 \beta l| e^{-2 \alpha l}$ is power transmission function, depending on $\theta_{i}$ and the distance $l$ in tires.

When a plane EM wave incident at an oblique angle on tires interface, there are two cases to be considered: incident electric field has polarization parallel to the plane of incidence, and incident electric field has polarization that is perpendicular to the plane of incidence. So, from the boundary conditions, that is, continuity of tangential electric and magnetic fields at the car tire interface, and using the Snell's laws of reflection and refraction, the $T_{c}$ can be derived as follows.

Case 1. Parallel polarization:

$$
T_{c}=\frac{2 \eta_{1} \cos \theta_{i}}{\eta_{1} \cos \theta_{t}+\eta_{0} \cos \theta_{i}} .
$$

Case 2. Perpendicular polarization:

$$
T_{c}=\frac{2 \eta_{1} \cos \theta_{i}}{\eta_{1} \cos \theta_{i}+\eta_{0} \cos \theta_{t}} .
$$

\section{Numerical Analyses}

5.1. Measuring the Permittivity of Tires. A standard tire (CPC2205/55 R16 91V) with the known permittivity (the permittivity is $\varepsilon^{\prime}=3.5$ and $\tan \delta=0.03$ at frequency $866 \mathrm{MHz}$ ) is used to determine the parameters $C_{i}, C_{0}, C_{1}$, and $\mathrm{C}_{2}$. Using the measuring methods presented in Section 2.1, $C_{i}, C_{0}, C_{1}$, and $C_{2}$ are $0.00113 \mathrm{pF}, 2.324 \times 10-14 \mathrm{pF}, 1.518 \times 10-$ $42 \mathrm{pF}$, and $1.6345 \times 10-50 \mathrm{pF}$, respectively.

The measuring results for reflection coefficients in a Smith chart are shown in Figure 7, using a test car tire (GL 274A, made in Guizhou Tire Co., LTD., China) with a vector network analyzer (AV 3629/A) between $100 \mathrm{MHz}$ and $5 \mathrm{GHz}$ at room temperature. Using (4) and (5), the permittivity of the test car tire can be determined to be $\varepsilon^{\prime}=3.78$ and $\tan \delta=0.038$.

Measuring and simulation results can be compared using the methods presented in Section 2.3. In the OCP model, the inner and outer radiuses of the probe are $0.65 \mathrm{~mm}$ and $2.35 \mathrm{~mm}$, respectively. The thickness of Teflon with a relative permittivity of $\mathcal{\varepsilon}^{\prime}$ and $\tan \delta=0.001$ is $1.35 \mathrm{~mm}$. The test tire is modeled as a cylinder with a radius of $10 \mathrm{~mm}$ and a height of $10 \mathrm{~mm}$. The absolute error of the real part of simulation and measuring coefficients and the absolute

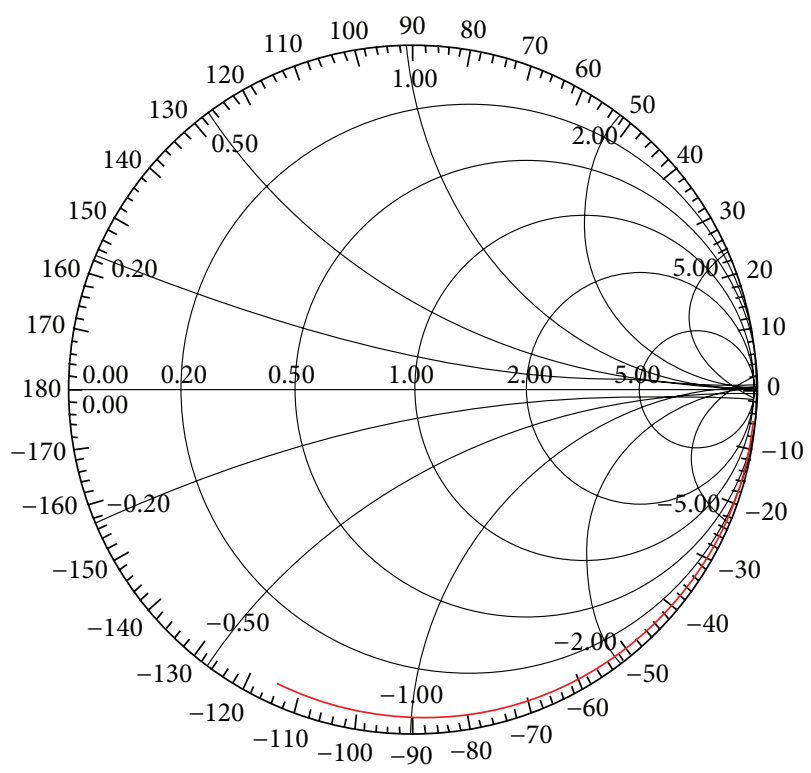

FIGURE 7: Measured reflection coefficients of a test car tire.

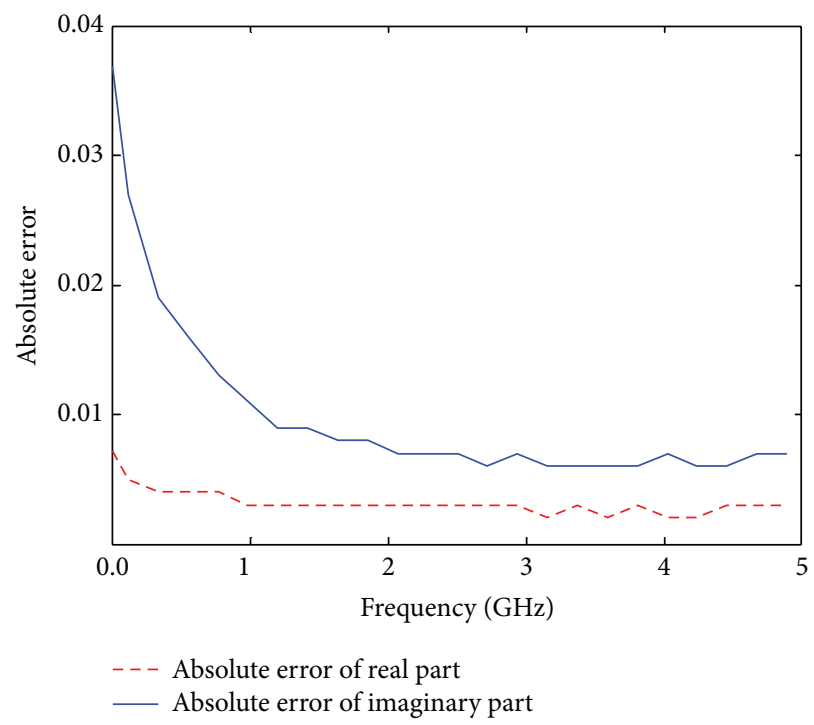

FIGURE 8: Absolute error values of real part and imaginary part.

error of the imaginary part of these versus frequency are shown in Figure 8. And the absolute error of the simulation and measuring coefficients is shown in Figure 9. Figures 8 and 9 show that there is small deviation $(<0.004)$ between simulation and measurement. So, it shows that the numerical simulation has good agreement with physical experiment, and the methods measuring the permittivity of tires are feasible.

5.2. Effects of Incident Angle $\theta_{i}$ on $T_{c}$. The permittivity of the test tire is $\varepsilon^{\prime}=3.78$ and $\tan \delta=0.038$ at frequency $866 \mathrm{MHz}$ from Section 5.1. The incident angle $\theta_{i}$ is $0^{\circ} \sim 90^{\circ}$. A magnitude of $T_{c}$ for the parallel and perpendicular polarization is shown in Figure 10. 


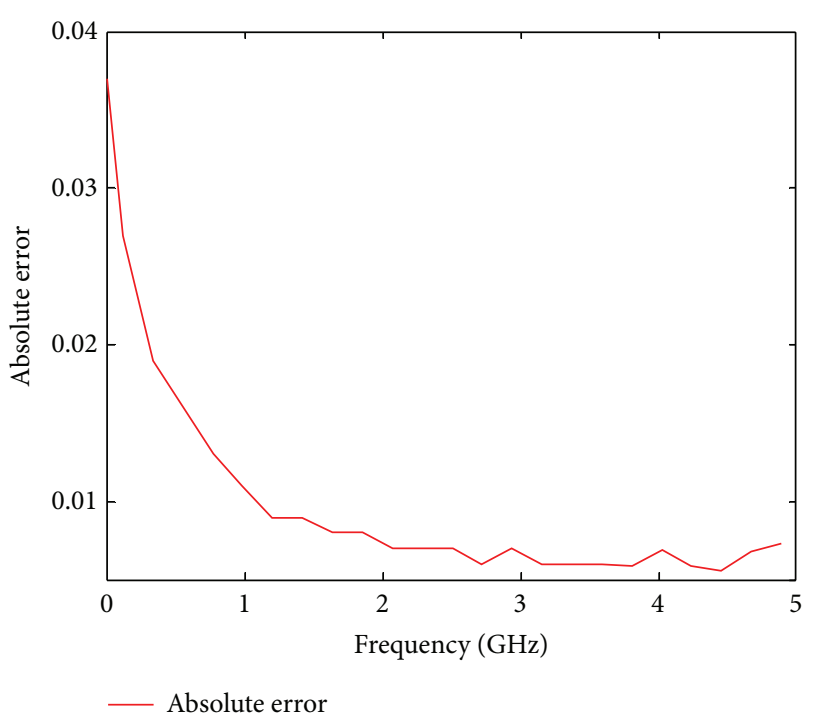

FIGURE 9: Absolute error values.

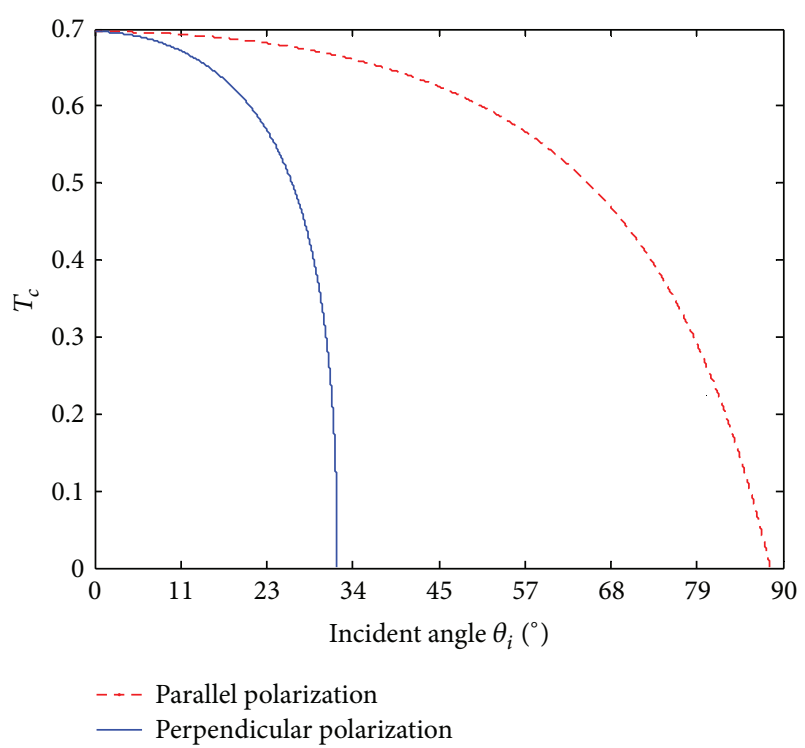

Figure 10: Effects of $\theta_{i}$ on $T_{c}$.

In Figure 10, $T_{c}$ for the parallel and perpendicular polarization decreases with increasing incident angle $\theta_{i} . T_{c}$ for the perpendicular polarization decreases faster than $T_{c}$ for the parallel polarization. This conclusion can be obtained from the experiment in Figure 11. The experiment setup contains a reader XC-RF807 and two liner polarization antennae XCAF26 (one is a horizontal polarization antenna and the other one is vertical polarization). The carrier frequency of the reader is $866 \mathrm{MHz}$, and the power o is $30 \mathrm{dBmW}$. The gain of the antenna is greater than $12 \mathrm{dBi}$, and the frequency range is from $840 \mathrm{MHz}$ to $868 \mathrm{MHz}$. The distance between GL274A tire and reader is $1 \mathrm{~m}$, and the depth of the tag embed in the tire is $9 \mathrm{~mm}$. Figure 12 shows the relation between the identification rate and angle of incidence.

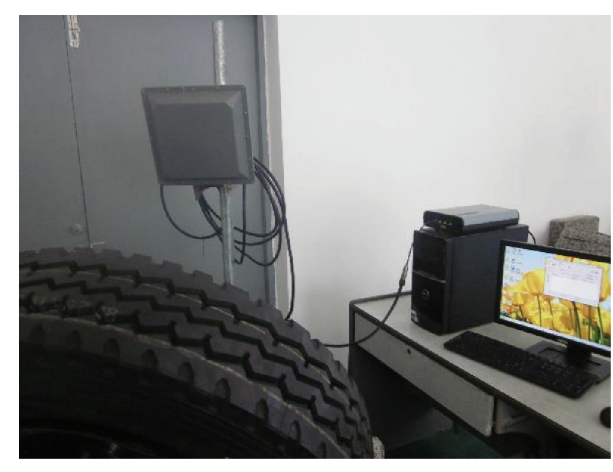

FIGURE 11: Experiment setup.

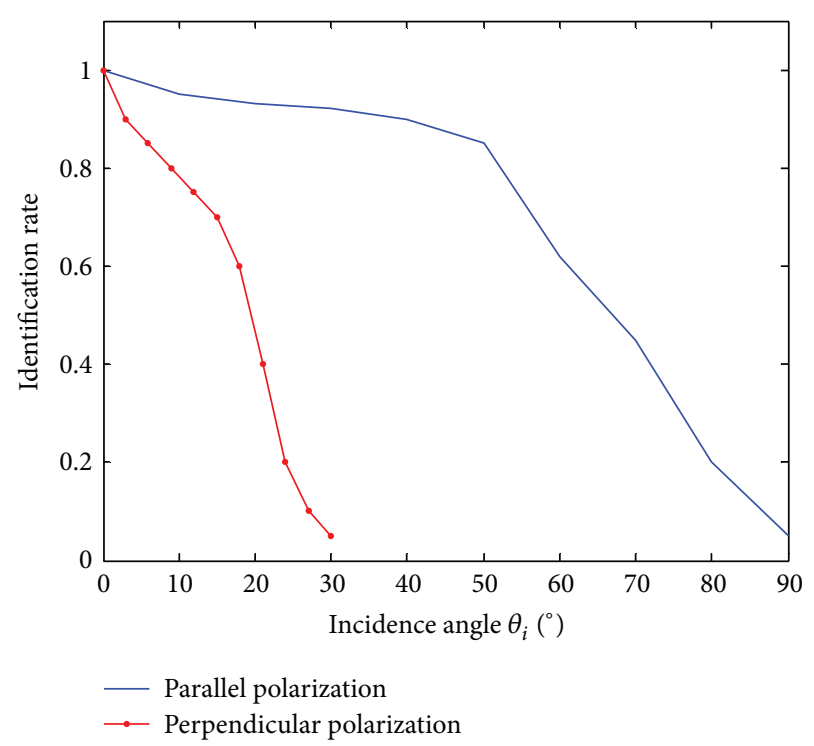

FIGURE 12: Relations between angle of incidence $\theta_{i}$ and the identification rate.

So, the parallel polarization and normal incidence are chosen for improving the performance of UHF RFID embedded in tires.

5.3. Effects of Distance l on $T\left(f, \theta_{i}, l\right)$. For convenience, The incident angle $\theta_{i}$ is $0^{\circ}$, and the permittivity of the test tire is $\varepsilon^{\prime}=3.78$ and $\tan \delta=0.038$ at frequency $866 \mathrm{MHz}$ f. A magnitude of $T\left(f, \theta_{i}, l\right)$ for the distance $l$ is shown in Figure 13.

In Figure 13, $T\left(f, \theta_{i}, l\right)$ decreases periodically with increasing the distance $l$ in tires. This conclusion can be obtained from the experiment in Figure 11. In this experiment, 8 GAL274A tires are used from Guizhou tire limited company in China. When the tires are vulcanizing, the depths of the tag embedded in the tire are $0.0 \mathrm{~mm}$ (tag is pasted on the surface of the tire), $3.0 \mathrm{~mm}, 6.0 \mathrm{~mm}, 9.0 \mathrm{~mm}$, $12 \mathrm{~mm}, 15 \mathrm{~mm}, 18 \mathrm{~mm}, 21 \mathrm{~mm}$, and $24 \mathrm{~mm}$, respectively (because of the limitation of the thickness of the tire, the biggest embedded depth is $24 \mathrm{~mm}$ ). Figure 14 shows the relation between the recognition rate and the embedded depth $l$. It means the recognition rate of tire embedded RFID 


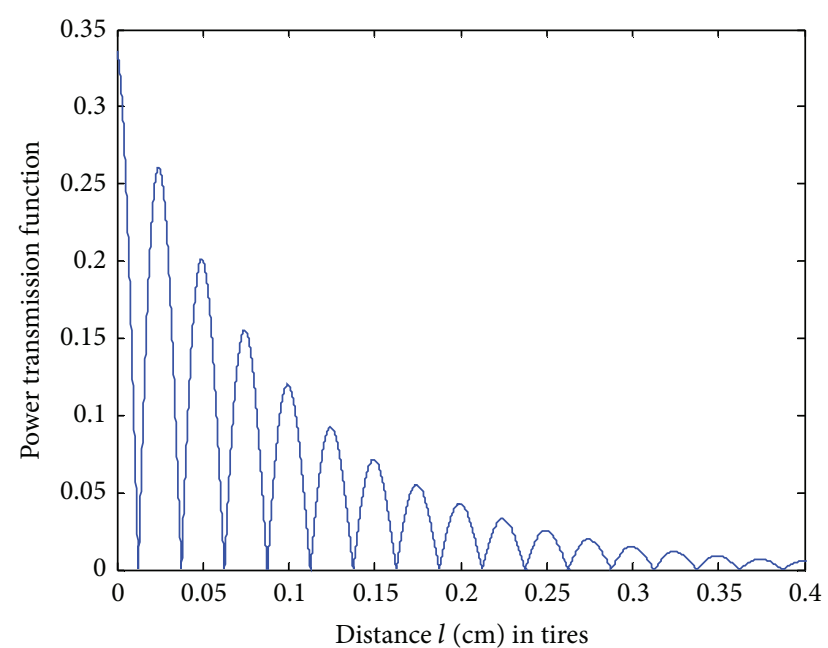

Figure 13: Effects of $l$ on $T\left(f, \theta_{i}, l\right)$.

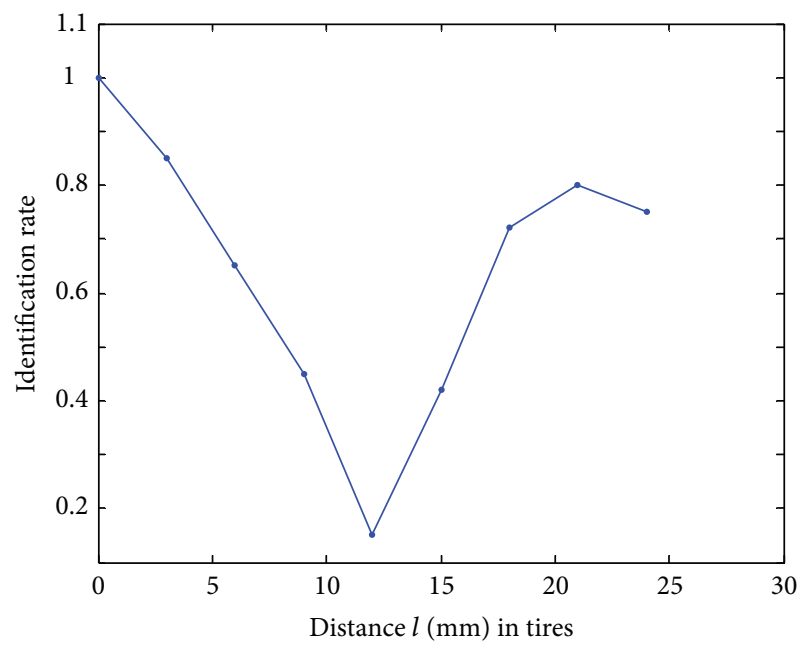

FIGURE 14: Relations $l$ and identification rate.

system declines along with $l$ also fluctuates along with $l$. This means the power transmission of tire embedded RFID system declines along with $l$ also fluctuates along with $l$.

So, the distance $l$ is chosen to keep $T\left(f, \theta_{i}, l\right)$ from locating valley.

\section{Conclusions}

From now on, UHF RFID tags embedded in tires have a deep impact on tire life cycle management and tire monitoring. So, we present the power transmission of wave propagation for UHF embedded RFID in tires.

In UHF passive embedded RFID systems in tires, the bidirectional radio link between reader and tags goes through air and tires. The total path loss contains reflection loss at tireair boundaries. So, we give the OCP method for measuring the permittivity of tires. By analyzing the radio link for UHF passive RFID, we establish a model of wave propagation of
UHF embedded RFID in tires and make numerical analyses. Finally, we make the conclusion as follows.

(i) The error of the OCP methods for measuring the permittivity of tires is small. And the methods can be used in measuring the permittivity of tires for designing the UHF embedded RFID in tires.

(ii) It is necessary to optimize and design the antenna of tag for the impedance matching of tag antenna and chip. This can improve the performance of the UHF embedded RFID in tires.

(iii) The parallel polarization and normal incidence are chosen for improving the performance of the UHF embedded RFID in tires. Finally, the distance $l$ is chosen to keep $T\left(f, \theta_{i}, l\right)$ from locating valley.

\section{Conflict of Interests}

The authors declare that they have no financial and personal relationships with other people or organizations that can inappropriately influence their work; there is no professional or other personal interests of any nature or kind in any product, service, and/or company that could be construed as influencing the position presented in this paper.

\section{Acknowledgments}

The authors wish to thank the editor and reviewers for their valuable comments, corrections, and suggestions, which led to an improved version of the original paper. This research is a project partially supported by the Guizhou Natural Science Foundation (Grant no. 2012[38]), the National Natural Science Foundation of China (Grant no. 61362004), and Guizhou Science and Technology Innovation Group for RFID \& WSN.

\section{References}

[1] S. Basat, K. Lim, I. Kim, M. M. Tentzeris, and J. Laskar, "Design and development of a miniaturized embedded UHF RFID tag for automotive tire applications," in Proceedings of the 55th Electronic Components and Technology Conference (ECTC '05), pp. 867-870, Lake Buena Vista, Fla, USA, June 2005.

[2] RFID 24-7, USA, "Michelin rolls out RFID-enabled tires for London Olympic," 1 pages, 2012, http://www.rfid24-7.com/ article/michelin-rolls-out-rfid-enabled-tires-for-london-olympics/.

[3] RFID Journal, USA, "Michelin embeds RFID tags in tires," 2 pages, 2012, http://www.rfidjournal.com/article/view/269/1/1.

[4] RFID Journal, USA, "RFID chip to monitor tire pressure," 2 pages, 2012, http://www.rfidjournal.com/article/view/93/1/1.

[5] J. D. Griffin and G. D. Durgin, "Complete link budgets for backscatter-radio and RFID systems," IEEE Antennas and Propagation Magazine, vol. 51, no. 2, pp. 11-25, 2009.

[6] P. V. Nikitin and K. V. S. Rao, "Antennas and propagation in UHF RFID systems," in Proceedings of the IEEE International Conference on RFID (IEEE RFID '08), pp. 277-288, Las Vegas, Nev, USA, April 2008. 
[7] P. V. Nikitin and K. V. S. Rao, "Theory and measurement of backscattering from RFID tags," IEEE Antennas and Propagation Magazine, vol. 48, no. 6, pp. 212-218, 2006.

[8] V. Komarov, S. Wang, and J. Tang, "Permittivity and measurement," in The Wiley Encyclopedia of RF and Microwave Engineering, vol. 4, pp. 3693-3711, 2005.

[9] C. C. Courtney, "Time-domain measurement of the electromagnetic properties of materials," IEEE Transactions on Microwave Theory and Techniques, vol. 46, no. 5, pp. 517-522, 1998.

[10] S. Jing, D. Ding, and Q. Jing, "Measurement of electromagnetic properties of materials using transmission/reflection method in coaxial line," in Proceedings of the 3rd Asia Pacific Conference on Environmental Electromagnetics, pp. 129-135, Hangzhou, China, 2003.

[11] D. K. Misra, "A quasi-static analysis of open-ended coaxial lines," IEEE Transactions on Microwave Theory and Techniques, vol. 35, no. 10, pp. 925-928, 1988.

[12] J. Grosinger and A. L. Scholtz, "Antennas and wave propagation in novel wireless sensing applications based on passive UHF RFID," Elektrotechnik und Informationstechnik, vol. 128, no. 1112, pp. 408-414, 2011.

[13] A. V. Hippel, Dielectrics and Waves, John Wiley \& Sons, New York, NY, USA, 1954. 

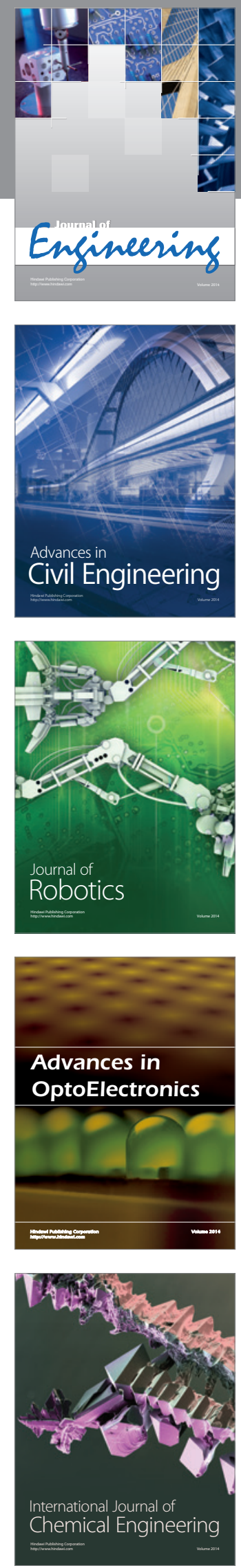

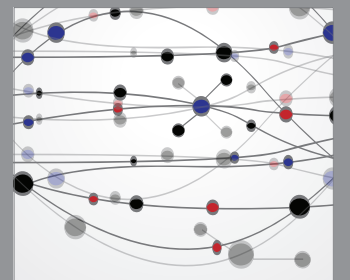

The Scientific World Journal
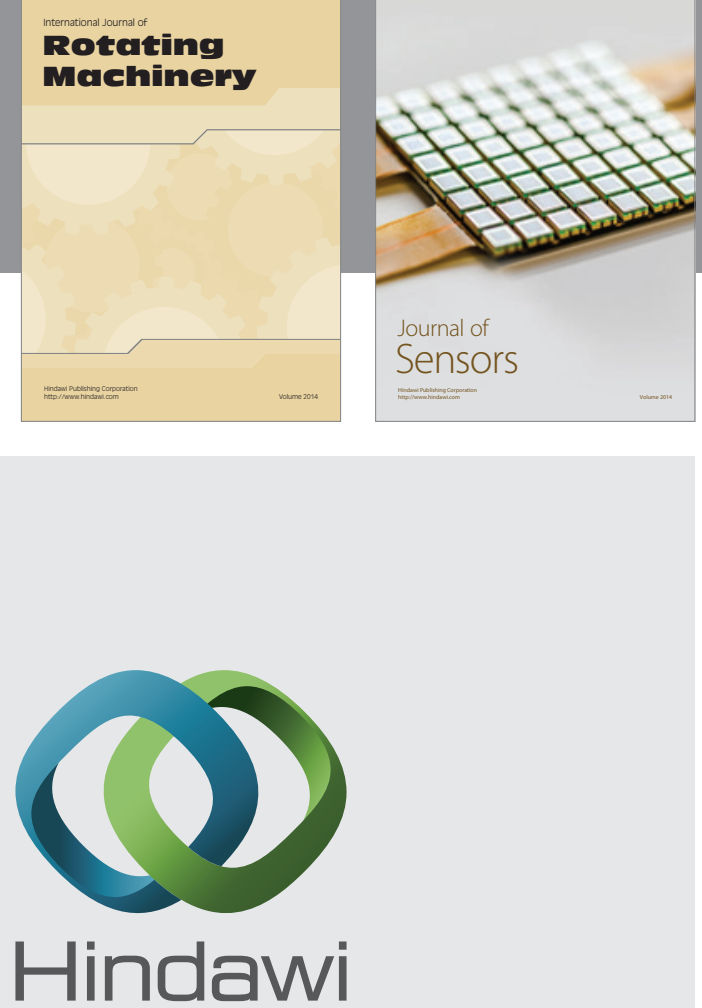

Submit your manuscripts at http://www.hindawi.com
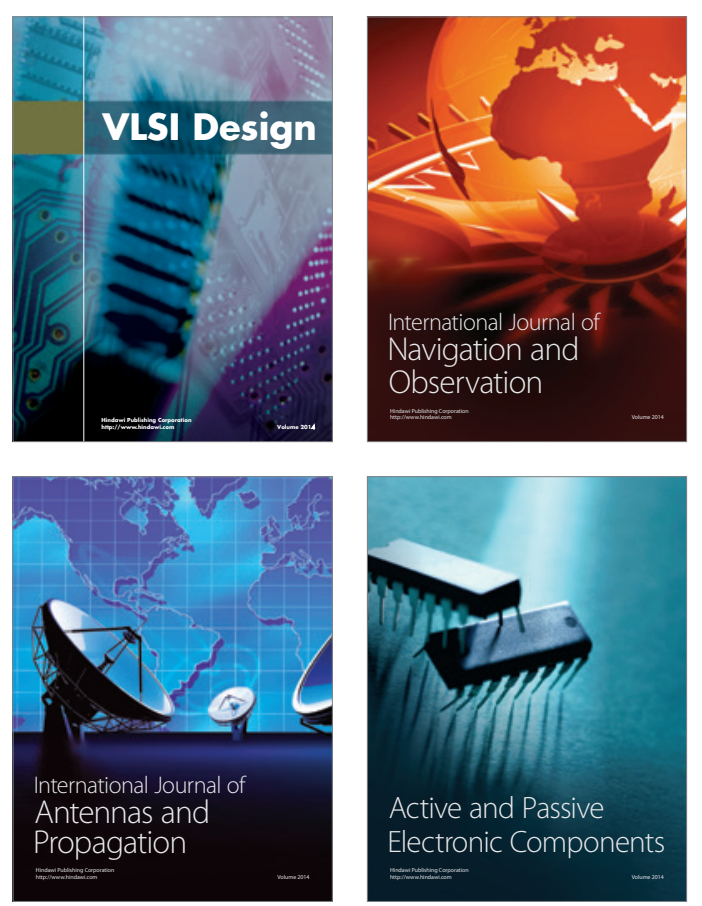
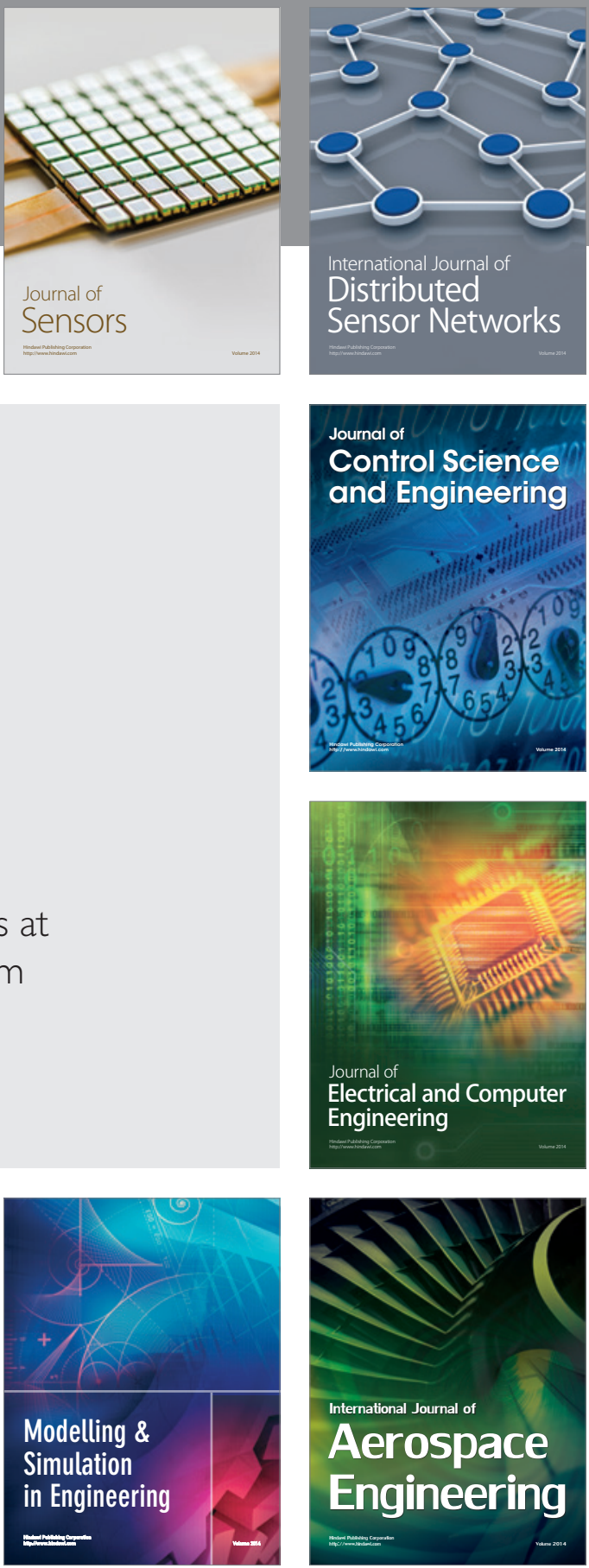

Journal of

Control Science

and Engineering
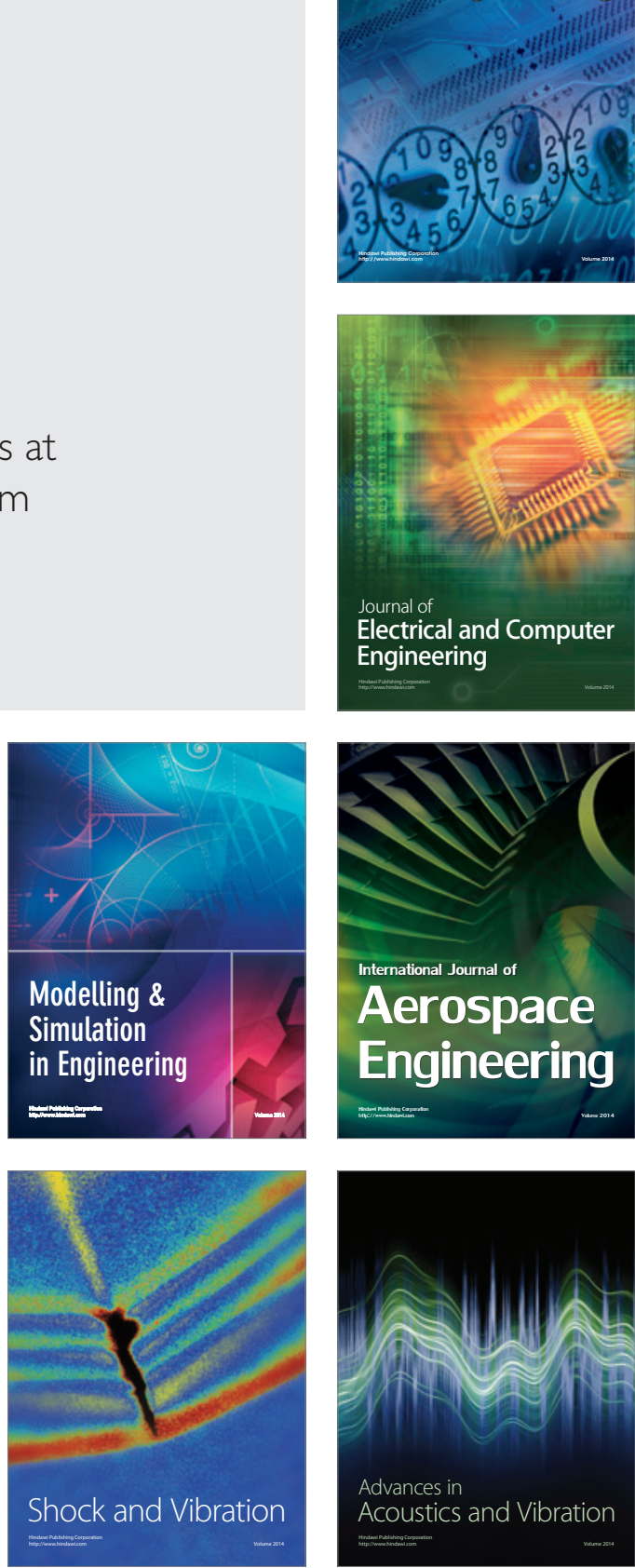